Christoffer Wenzel Tornøe · Judith L. Jacobsen · Henrik Madsen

\title{
Grey-box pharmacokinetic/pharmacodynamic modelling of a euglycaemic clamp study
}

Received: 21 January 2003 / Revised version: 12 August 2003 /

Published online: 2 December 2003 - (C) Springer-Verlag 2003

\begin{abstract}
Grey-box pharmacokinetic/pharmacodynamic (PK/PD) modelling is presented as a promising way of modelling the pharmacokinetics and pharmacodynamics of the in vivo system of insulin and glucose and to estimate model and derived PK/PD parameters. The concept behind grey-box modelling consists in using a priori physiological knowledge along with information from data in the estimation of model parameters. The PK/PD properties of two types of insulin are investigated in a euglycaemic clamp study where a single bolus of insulin is injected subcutaneously. The effect of insulin on the glucose disappearance is investigated by artificially maintaining a blood glucose concentration close to the normal fasting level. The infused glucose needed to maintain the clamped blood glucose concentration can therefore be used as a measure for the glucose utilization. The PK and PD parameters are successfully estimated simultaneously thereby describing the uptake, distribution, and effect of the two different types of insulin.
\end{abstract}

\section{Introduction}

The aim of the present paper is to model the in vivo dynamical system of insulin and glucose from a euglycaemic clamp study using grey-box pharmacokinetic/pharmacodynamic (PK/PD) modelling. This consists in using a plausible PK/PD model structure combined with a stochastic term representing unmodelled dynamics of the system, inputs to the system which are not measured, and disturbances. The grey-box modelling concept is a very attractive approach as the insulin/glucose dynamics is not fully understood or cannot be explicitly modelled. First of all, the approach enables a possibility for combining the physiological knowledge with information from data. Secondly, the parameters in the models have physical meaning and therefore more readily may be interpreted. Furthermore, it is possible to treat missing data and to model non-linear (NL) and time-varying systems.

The euglycaemic clamp study is used to determine the characteristics of different types of insulin, their absorption, distribution, and elimination kinetics along with its pharmacodynamic characteristics. The information obtained from clamp

C.W. Tornøe, H. Madsen: Informatics and Mathematical Modelling, Technical University of Denmark, Building 321, 2800 Lyngby, Denmark

\section{J.L. Jacobsen: Department of Biostatistics, Novo Nordisk A/S, 2880 Bagsværd, Denmark}

C.W. Tornøe: Ferring Pharmaceuticals A/S, Kay Fiskers Plads 11, 2300 Copenhagen S, Denmark. e-mail: christoffer.tornoe@ferring.com

Key words or phrases: Grey-box modelling - Compartmental modelling - Insulin - Euglycaemic clamp - Stochastic differential equations - ML estimation - Kalman filtering 
studies is usually used in phase I clinical trials where the insulin is tested in healthy volunteers to verify that it has the intended properties without unwanted side effects and to determine the insulin dose needed to keep the individual in metabolic control.

\section{Experimental procedures}

The euglycaemic clamp study is performed on twenty healthy non-smoking Caucasian males between 18 and 40 years of age with a body mass index less than $27 \mathrm{~kg} / \mathrm{m}^{2}$.

The experimental design is a single center two-period randomized double blind crossover experiment. Each patient receives a bolus dose of either a human insulin analogue (Insulin Aspart, NovoRapid ${ }^{\circledR}$ ) or a faster acting insulin (Actrapid ${ }^{\circledR}$ ) on the first day of the study and the other type of insulin at the next visit which is one to two weeks later to prevent carry-over effects from the first treatment to the next. From here on, NovoRapid ${ }^{\circledR}$ is referred to by insulin A and Actrapid ${ }^{\circledR}$ by insulin B.

The insulin and C-peptide levels are measured by inserting a catheter into an antecubital vein in the patient's arm. The glucose concentration is measured through a glucose sensor in the hand of the same arm. To suppress the secretion of insulin from the pancreas, the patient continuously receives an IV infusion of regular human insulin $(0.15 \mathrm{mU} / \mathrm{kg} \mathrm{BW} / \mathrm{min})$ during the whole experiment in the opposite arm along with an IV infusion of glucose (GIR) to maintain a constant blood glucose (BG) concentration. The experiment can be thought of as a kind of titration where the amount of infused glucose needed to maintain euglycemia (constant glucose concentration) can be assumed to be equal to the amount of glucose utilized in the body.

After 90 min. of monitoring the insulin and the BG, the patient receives a single bolus dose of either insulin A or B $(0.2 \mathrm{U} / \mathrm{kg} \mathrm{BW})$ which is injected subcutaneously (SC) and the insulin, GIR, BG, and C-peptide concentration are observed during the next 10 hours.

The plasma insulin concentration is sampled at non-equidistant time instants. Each patient is monitored $90 \mathrm{~min}$. before the injection with samples every $30 \mathrm{~min}$. until the time of injection. The patients are thereafter monitored for 10 hours with samples every $10 \mathrm{~min}$. during the first hour, every $15 \mathrm{~min}$. the next hour, and every 20 min. the last 8 hours. The GIR, BG, and C-peptide concentration are sampled every minute throughout the entire study.

The plasma insulin concentration, GIR, and BG concentration are shown in Fig. 1 for a representative subject from the study.

\section{Statistical methods}

The statistical framework behind grey-box modelling is briefly introduced in this section. The equations used in grey-box models for describing the dynamics are stochastic differential equations defined as

$$
d \boldsymbol{x}_{t}=\boldsymbol{f}\left(\boldsymbol{x}_{t}, \boldsymbol{u}_{t}, \boldsymbol{\theta}, t\right) d t+\boldsymbol{G}\left(\boldsymbol{x}_{t}, \boldsymbol{u}_{t}, \boldsymbol{\theta}, t\right) d \boldsymbol{w}_{t}
$$




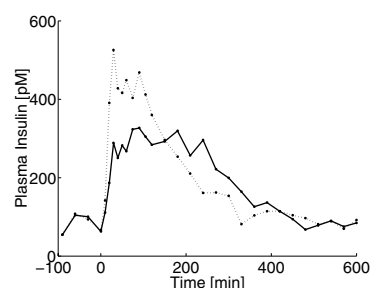

(a) Insulin

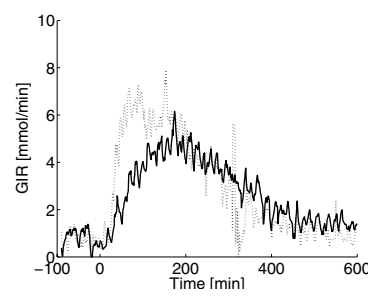

(b) GIR

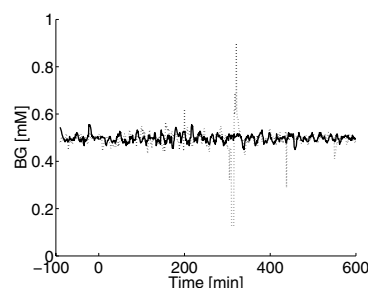

(c) $\mathrm{BG}$

Fig. 1. Plasma insulin, GIR, and BG concentration for a representative subject for treatment with insulin A (solid line) and insulin B (dotted line).

where $\boldsymbol{x}_{t}$ is the state vector, $\boldsymbol{u}_{t}$ is the input vector, $\boldsymbol{\theta}$ is the parameter vector, $\boldsymbol{f}$ is a deterministic function called the drift term, $\boldsymbol{G}$ is the diffusion term, and $\boldsymbol{w}_{t}$ is a standard Wiener process. The Wiener process is a non-stationary stochastic process that starts at 0 and has mutually independent (orthogonal) increments which are normally distributed with mean and covariance [6]

$$
\begin{aligned}
& E\left[\boldsymbol{w}_{t}-\boldsymbol{w}_{s}\right]=0 \\
& V\left[\boldsymbol{w}_{t}-\boldsymbol{w}_{s}\right]=\sigma^{2}|t-s|
\end{aligned}
$$

The derivative of the Wiener process has a constant spectral density for all frequencies and thus has infinite variance. This makes it the closest to the concept 'continuous white noise' [6]. A more detailed description of the theory behind stochastic differential equations can be found in [8].

The relationship between input and output signals in a dynamical system is conveniently described by a state space model. A state space model is an internal parametric representation between input and output which in a continuous time formulation enables a direct physical meaning of the parameters. Since the structural information of the physical system is formulated in continuous time and the data is observed at discrete time, the following continuous-discrete time stochastic state space model, consisting of a continuous time system equation and a discrete time observation equation, is used.

$$
\begin{aligned}
d \boldsymbol{x}_{t} & =\boldsymbol{f}\left(\boldsymbol{x}_{t}, \boldsymbol{u}_{t}, \boldsymbol{\theta}, t\right) d t+\boldsymbol{G}\left(\boldsymbol{x}_{t}, \boldsymbol{u}_{t}, \boldsymbol{\theta}, t\right) d \boldsymbol{w}_{t} \\
\boldsymbol{y}_{k} & =\boldsymbol{h}\left(\boldsymbol{x}_{k}, \boldsymbol{u}_{k}, \boldsymbol{\theta}, t_{k}\right)+\boldsymbol{e}_{k}
\end{aligned}
$$

where $\boldsymbol{y}_{k}$ is a vector of the measurements at time $t_{k}$.

The observation equation (5) describes what is actually measured at discrete time instants $t_{k}$, and $\boldsymbol{y}_{k}$ is a function of the state $\boldsymbol{x}_{t}$ contaminated with Gaussian distributed white noise $\boldsymbol{e}_{k}$. The system noise $\boldsymbol{w}_{t}$ and observation noise $\boldsymbol{e}_{k}$ are assumed mutually independent.

The two most widely used methods for parameter estimation in continuous time state space models are: Maximum likelihood (ML) and maximum a posteriori (MAP) estimation. The major difference between these two approaches is that MAP estimation uses not only the experimental data, but also a priori available information on the parameter vector (Baysian approach), e.g. prior mean and covariance 
matrix in the Gaussian case, while for the ML approach, only experimental measurements are used. In the following, only ML estimation is considered since no a priori information is available for the modelled system.

Maximum likelihood estimation is based on maximizing the likelihood function of the observations $\mathcal{Y}_{N}=\left[\boldsymbol{y}_{N}, \boldsymbol{y}_{N-1}, \ldots, \boldsymbol{y}_{0}\right]$ given the parameter vector $\boldsymbol{\theta}$ [5].

The likelihood function is [6]

$$
\begin{aligned}
L\left(\boldsymbol{\theta} ; \mathcal{Y}_{N}\right)=p\left(\mathcal{Y}_{N} \mid \boldsymbol{\theta}\right) & =p\left(\boldsymbol{y}_{N} \cap \mathcal{Y}_{N-1} \mid \boldsymbol{\theta}\right) \\
& =p\left(\boldsymbol{y}_{N} \mid \mathcal{Y}_{N-1}, \boldsymbol{\theta}\right) p\left(\mathcal{Y}_{N-1} \mid \boldsymbol{\theta}\right) \\
& =\left(\prod_{k=1}^{N} p\left(\boldsymbol{y}_{k} \mid \mathcal{Y}_{k-1}, \boldsymbol{\theta}\right)\right) p\left(\boldsymbol{y}_{0} \mid \boldsymbol{\theta}\right)
\end{aligned}
$$

where the Markov property $P(A \cap B)=P(A \mid B) P(B)$ has been applied to express the likelihood function as a product of marginal conditional probability densities. Under the assumption that the conditional density function is Gaussian, which is true for linear models, the following equations characterizes entirely the one-step conditional density $p\left(\boldsymbol{y}_{k} \mid \mathcal{Y}_{k-1}, \boldsymbol{\theta}\right)$ in (6).

$$
\begin{aligned}
\hat{\boldsymbol{y}}_{k \mid k-1} & =E\left\{\boldsymbol{y}_{k} \mid \mathcal{Y}_{k-1}, \boldsymbol{\theta}\right\} \\
\boldsymbol{R}_{k \mid k-1} & =V\left\{\boldsymbol{y}_{k} \mid \mathcal{Y}_{k-1}, \boldsymbol{\theta}\right\}
\end{aligned}
$$

By introducing the one-step prediction error or innovation

$$
\boldsymbol{\epsilon}_{k}=\boldsymbol{y}_{k}-\hat{\boldsymbol{y}}_{k \mid k-1}
$$

the likelihood function can then be written as

$$
L\left(\boldsymbol{\theta} ; \mathcal{Y}_{N}\right)=\left(\prod_{k=1}^{N} \frac{\exp \left(-\frac{1}{2} \boldsymbol{\epsilon}_{k}^{T} \boldsymbol{R}_{k \mid k-1}^{-1} \boldsymbol{\epsilon}_{k}\right)}{\sqrt{\operatorname{det}\left(\boldsymbol{R}_{k \mid k-1}\right)}(\sqrt{2 \pi})^{l}}\right) p\left(\boldsymbol{y}_{0} \mid \boldsymbol{\theta}\right)
$$

where $l$ is the dimension of $\boldsymbol{y}_{k}$.

If the likelihood function is further conditioned on $\boldsymbol{y}_{0}$ and by taking the logarithm of (10), the following equation is obtained.

$$
\begin{aligned}
-\ln \left(L\left(\boldsymbol{\theta} ; \mathcal{Y}_{N} \mid \boldsymbol{y}_{0}\right)\right)= & \frac{1}{2} N l \ln (2 \pi)+\frac{1}{2} \sum_{k=1}^{N}\left(\ln \left(\operatorname{det}\left(\boldsymbol{R}_{k \mid k-1}\right)\right)\right. \\
& \left.+\boldsymbol{\epsilon}_{k}^{T} \boldsymbol{R}_{k \mid k-1}^{-1} \boldsymbol{\epsilon}_{k}\right)
\end{aligned}
$$

The innovation $\boldsymbol{\epsilon}_{k}$ and the conditional covariance $\boldsymbol{R}_{k \mid k-1}$ can be calculated for given parameters $\boldsymbol{\theta}$ and initial conditions $\boldsymbol{x}_{0}$ by the use of the Kalman Filter (KF) or the Extended Kalman Filter (EKF) for linear and non-linear systems, respectively. The ML estimate of the parameters $\boldsymbol{\theta}$ are now found as

$$
\hat{\boldsymbol{\theta}}=\arg \min _{\boldsymbol{\theta} \in \boldsymbol{\Theta}}\left\{-\ln \left(L\left(\boldsymbol{\theta} ; \mathcal{Y}_{N} \mid \boldsymbol{y}_{0}\right)\right)\right\}
$$


The maximum likelihood estimates are asymptotically normally distributed with mean $\boldsymbol{\theta}$ and a covariance matrix $\boldsymbol{D}$ found from the Fisher information matrix [6]. An approximation of $\boldsymbol{D}$ can be found by

$$
\boldsymbol{D}(\hat{\boldsymbol{\theta}}) \simeq \boldsymbol{H}^{-1}
$$

where $\boldsymbol{H}$ is the Hessian calculated as the second derivative of the negative log-likelihood function (11) at the obtained parameter estimate.

State filtering is introduced as a tool for estimating the underlying states at time $t$ based on the measurements until time $t_{k}\left(t \geq t_{k}\right)$.

The KF is a set of mathematical equations that provides an efficient and exact recursive approach to calculate the conditional mean (7) and covariance (8) for linear systems. In the following, the KF will be given for the following linear time-invariant (LTI) state space model [6].

$$
\begin{aligned}
d \boldsymbol{x}_{t} & =\left(\boldsymbol{A}(\boldsymbol{\theta}) \boldsymbol{x}_{t}+\boldsymbol{B}(\boldsymbol{\theta}) \boldsymbol{u}_{t}\right) d t+\boldsymbol{\sigma}(\boldsymbol{\theta}) d \boldsymbol{w}_{t} \\
\boldsymbol{y}_{k} & =\boldsymbol{C}(\boldsymbol{\theta}) \boldsymbol{x}_{k}+\boldsymbol{D}(\boldsymbol{\theta}) \boldsymbol{u}_{k}+\boldsymbol{e}_{k}
\end{aligned}
$$

where $\boldsymbol{A}, \boldsymbol{B}, \boldsymbol{C}$, and $\boldsymbol{D}$ are constant coefficient matrices, $\boldsymbol{w}_{t}$ is a standard Wiener process assumed to be mutually independent of $\boldsymbol{e}_{k}$, which is a Gaussian white noise process with zero mean and covariance $\boldsymbol{S}\left(\boldsymbol{\theta}, t_{k}\right)$.

The update equations of the KF are

$$
\begin{aligned}
& \hat{\boldsymbol{x}}_{k \mid k}=\hat{\boldsymbol{x}}_{k \mid k-1}+\boldsymbol{K}_{k}\left(\boldsymbol{y}_{k}-\hat{\boldsymbol{y}}_{k \mid k-1}\right) \\
& \boldsymbol{P}_{k \mid k}=\boldsymbol{P}_{k \mid k-1}-\boldsymbol{K}_{k} \boldsymbol{R}_{k \mid k-1} \boldsymbol{K}_{k}^{T}
\end{aligned}
$$

The initial conditions are $\hat{\boldsymbol{x}}_{1 \mid 0}=\mu_{0}$ and $\boldsymbol{P}_{1 \mid 0}=\boldsymbol{V}_{0} \cdot \hat{\boldsymbol{x}}_{k \mid k}$ is the estimate of the state, $\boldsymbol{P}_{k \mid k}$ is the associated covariance, and $\boldsymbol{K}_{k}$ is the Kalman gain given by

$$
\boldsymbol{K}_{k}=\boldsymbol{P}_{k \mid k-1} \boldsymbol{C}^{T} \boldsymbol{R}_{k \mid k-1}^{-1}
$$

Next, the state prediction equations, which are the optimal (minimum variance) linear prediction of the mean and covariance, can be calculated by solving

$$
\begin{aligned}
& d \hat{\boldsymbol{x}}_{t \mid k} / d t=\boldsymbol{A} \hat{\boldsymbol{x}}_{t \mid k}+\boldsymbol{B} \boldsymbol{u}_{t} \\
& d \boldsymbol{P}_{t \mid k} / d t=\boldsymbol{A} \boldsymbol{P}_{t \mid k}+\boldsymbol{P}_{t \mid k} \boldsymbol{A}^{T}+\boldsymbol{\sigma} \boldsymbol{\sigma}^{T}
\end{aligned}
$$

Using the solution to the stochastic differential equation (14), i.e.

$$
\boldsymbol{x}_{t_{k+1}}=e^{\boldsymbol{A}\left(t_{k+1}-t_{k}\right)} \boldsymbol{x}_{t_{k}}+\int_{t_{k}}^{t_{k+1}} e^{\boldsymbol{A}\left(t_{k+1}-s\right)} \boldsymbol{B} \boldsymbol{u}_{s} d s+\int_{t_{k}}^{t_{k+1}} e^{\boldsymbol{A}\left(t_{k+1}-s\right)} \boldsymbol{\sigma} d \boldsymbol{w}_{s}
$$

and assuming $\boldsymbol{u}$ to be constant during the sample interval $\left[t_{k}, t_{k+1}\right.$ [ the one-step prediction of the state mean and covariance are

$$
\begin{aligned}
\hat{\boldsymbol{x}}_{k+1 \mid k} & =E\left[\boldsymbol{x}_{t_{k+1}} \mid \boldsymbol{x}_{t_{k}}\right]=e^{\boldsymbol{A} \tau_{s}} \hat{\boldsymbol{x}}_{k \mid k}+\int_{0}^{\tau_{s}} e^{\boldsymbol{A} s} d s \boldsymbol{B} \boldsymbol{u}_{k} \\
\boldsymbol{P}_{k+1 \mid k} & =E\left[\boldsymbol{x}_{t_{k+1}} \boldsymbol{x}_{t_{k+1}}^{T} \mid \boldsymbol{x}_{t_{k}}\right] \\
& =e^{\boldsymbol{A} \tau_{s}} \boldsymbol{P}_{k \mid k}\left(e^{\boldsymbol{A} \tau_{s}}\right)^{T}+\int_{0}^{\tau_{s}} e^{\boldsymbol{A} s} \boldsymbol{\sigma} \boldsymbol{\sigma}^{T}\left(e^{\boldsymbol{A} s}\right)^{T} d s
\end{aligned}
$$


where $\tau_{s}=t_{k+1}-t_{k}$ is the sampling time. If $\boldsymbol{u}_{k}$ is not constant during the sampling interval other solutions exists - see [5].

Finally, the output prediction equations of the observation $\hat{\boldsymbol{y}}_{k+1 \mid k}$ along with the covariance $\boldsymbol{R}_{k+1 \mid k}$ are calculated by

$$
\begin{aligned}
\hat{\boldsymbol{y}}_{k+1 \mid k} & =\boldsymbol{C} \hat{\boldsymbol{x}}_{k+1 \mid k}+\boldsymbol{D} \boldsymbol{u}_{k+1} \\
\boldsymbol{R}_{k+1 \mid k} & =\boldsymbol{C} \boldsymbol{P}_{k+1 \mid k} \boldsymbol{C}^{T}+\boldsymbol{S}
\end{aligned}
$$

where $S$ is the covariance matrix of the measurement error.

The setup described above is implemented in the estimation program CTSM (Continuous Time Stochastic Modelling) for both linear and non-linear system. A detailed description about the ML and MAP estimation methods as well as the KF and EKF filtering techniques for linear and non-linear models, respectively, is given in the CTSM manual [5]. The application of stochastic differential equations in PK/PD modelling has previously been discussed in $[2,10,11]$.

\section{Compartmental model}

Several different PK models have been considered to account for the different SC absorption mechanism for the two types of insulin along with issues like NL elimination kinetics and tissue equilibration. But for the available plasma insulin measurements, it is found that the plasma insulin kinetics is adequately described by a single compartment representation. The different PK models which have been tested can be found in [13].

The insulin is assumed to be mixed instantaneously in the plasma. The actual time taken for mixing is approximately a few minutes and is therefore considered insignificant compared with the sampling time. The insulin absorption and elimination is assumed to follow first-order kinetics meaning that the rate of change of insulin concentration is directly proportional to the remaining concentration of insulin. This assumption leads to a linear model. The amount of insulin removed from the SC tissue is equal to the amount absorbed in the central compartment. This assumption is made because the break-down of SC insulin is not modelled. No insulin is assumed secreted from the pancreas because the IV infusion of regular human insulin suppresses the production. The small amount of insulin that actually is secreted in the pancreas is corrected by using C-peptide measurements. It is therefore reasonable not to include any feedback mechanisms in the model since they have been disrupted.

To determine which type of PK/PD model is needed to model the dynamics between insulin and glucose, a phase-plot of GIR vs. the plasma insulin concentration, where data points are connected in chronological order, is plotted in Fig. 2 for a representative subject from the study.

A counter-clockwise hysteresis loop is observed in the phase-plot since there exists two different values of GIR for any plasma insulin concentration depending on the time after the insulin administration. The delay for insulin A is smaller than that for insulin B since the hysteresis loop is smaller for insulin A. Had there been no hysteresis loop, a basic PK/PD model such as the single-compartment 


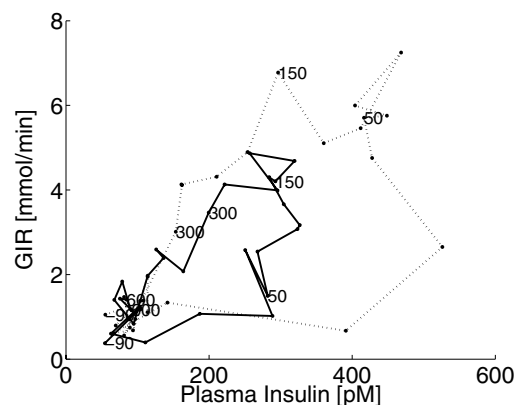

Fig. 2. Phase-plot of GIR vs. plasma insulin concentration for treatment with insulin A (solid line) and insulin B (dotted line). The numbers indicate the time after the insulin bolus injection.

model expanded with a direct link model could have been used. Instead, the effectcompartment model initially proposed by Sheiner et al. in [12] concerning its application to $d$-tubocurarine is used. The effect-compartment model has also previously been successfully applied to a similar euglycaemic clamp study in $[14,15]$.

The single-compartment PK model is expanded with a hypothetical effect compartment since the time course of insulin effect does not parallel the time course of insulin computed to reside in the central compartment. The effect site can be thought of as the extracellular space where the interaction with the biological receptor system takes place [9]. Modelling the kinetics of the effect site by adding an effect compartment is a simple way to correct non steady-state data to the equivalent of steady-state data so that a concentration-response curve can be discerned, unobscured by a hysteresis loop [7].

The effect-compartment model is illustrated in Fig. 3.

At steady-state, the concentration in the effect compartment $c_{e, s s}$ is equal to the concentration in the central compartment $c_{c, s s}$. The rate of input will therefore equal that of output, i.e. $k_{c e} \cdot q_{c}=k_{e 0} \cdot q_{e}$ [4]. This assumption allows for a calculation of the volume $V_{e}$ for the effect compartment by the following equation.

$$
V_{d} \cdot k_{c e} \cdot c_{c, s s}=V_{e} \cdot k_{e 0} \cdot c_{e, s s}
$$

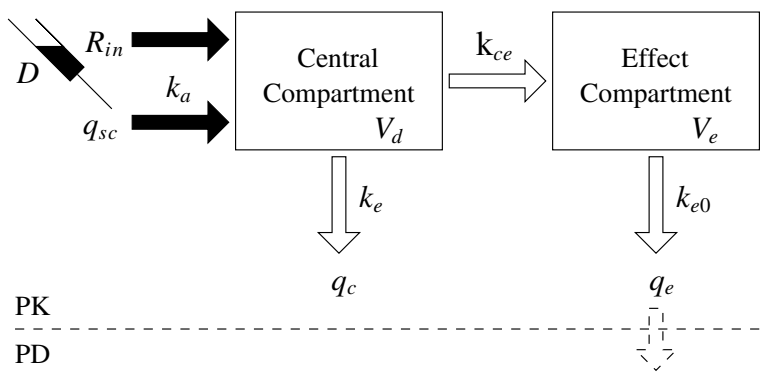

GIR

Fig. 3. Illustration of the effect-compartment model. 
The concentration in the effect compartment can then be calculated by dividing $q_{e}$ with $V_{e}$. When doing so, the rate constant for the irreversible elimination from the central compartment to the effect compartment $k_{c e}$ cancels out. The following system of equations thereby describes the PK part of the effect-compartment model.

$$
\begin{aligned}
\frac{d q_{s c}}{d t} & =D \cdot \delta(t)-k_{a} q_{s c} \\
\frac{d q_{c}}{d t} & =k_{a} q_{s c}+R_{i n}-k_{e} q_{c} \\
\frac{d c_{e}}{d t} & =K_{e 0}\left(\frac{q_{c}}{V_{d}}-c_{e}\right)
\end{aligned}
$$

where the effect compartment is assumed to receive a negligible mass from the central compartment, thereby not affecting the equations for the insulin in the central compartment.

The three states in the model are $q_{s c}$ which describes the amount of insulin remaining to be absorbed from the SC tissue $\left(q_{s c}=0\right.$ for $\left.t<0\right), q_{c}$ which represents the amount of insulin in the central compartment, and $c_{e}=q_{e} / V_{e}$ which is the insulin concentration in the effect compartment.

The parameters $k_{a}$ and $k_{e}$ are the rate constants for the irreversible absorption to and elimination from the central compartment, respectively. The parameters $V_{d}$ and $V_{e}$ are the central and effect compartment volumes. $V_{d}$ should not be mistaken with the plasma volume but can be thought of as the apparent volume of distribution in the body.

The time-dependent aspects of the equilibrium between the central and effect concentrations are only controlled by $K_{e 0}$ which is the equilibrium constant for the passive diffusion between the central and effect compartment.

The two inputs to the system are $D$ and $R_{i n} . D$ describes the SC injected insulin bolus dose of either type A or B given at time $t=0$ (as described by Dirac delta function $\delta(t))$ and $R_{i n}$ is the IV infusion of regular human insulin given throughout the study to suppress the secretion of insulin from the pancreas.

Because of the nature of the experimental procedure in the clamp study and since the endogenous production of insulin is ignored, the amount of infused glucose (GIR) needed to maintain euglycemia can be assumed to be equal to the amount of glucose utilized in the body. The GIR can thereby be used as the response to the injected insulin. The PD are therefore modelled by combining the GIR with the insulin concentration in the effect compartment using the Hill response equation (sigmoidal $E_{\text {max }}$ model), i.e.

$$
G I R=\frac{E_{\max }}{E C_{50}^{\gamma}+c_{e}^{\gamma}} \cdot c_{e}^{\gamma}
$$

where $E C_{50}$ is the insulin concentration producing $50 \%$ of the maximum effect $E_{\text {max }}$ while $\gamma$ is the sigmoidicity/response factor.

The equations governing the effect-compartment model can be written as the following continuous-discrete time state-space model where the three continuous time system equations are 


$$
\left[\begin{array}{c}
d q_{s c} \\
d q_{c} \\
d c_{e}
\end{array}\right]=\left[\begin{array}{ccc}
-k_{a} & 0 & 0 \\
k_{a} & -k_{e} & 0 \\
0 & K_{e 0} / V_{d} & -K_{e 0}
\end{array}\right]\left[\begin{array}{c}
q_{s c} \\
q_{c} \\
c_{e}
\end{array}\right] d t+\left[\begin{array}{ll}
1 & 0 \\
0 & 1 \\
0 & 0
\end{array}\right]\left[\begin{array}{c}
D \cdot \delta(t) \\
R_{i n}
\end{array}\right] d t+\boldsymbol{\sigma} d \boldsymbol{w}_{t}
$$

where $\boldsymbol{\sigma}$ is a diagonal matrix with $\sigma_{s c}, \sigma_{c}$, and $\sigma_{e}$ in the diagonal and $\boldsymbol{w}_{t}$ is a threedimensional Wiener process with independent components. The two discrete time observation equations can be written as

$$
\begin{aligned}
c_{I} & =\frac{q_{c}}{V_{d}}+e_{1, k} \\
G I R & =\frac{E_{\max }}{E C_{50}^{\gamma}+c_{e}^{\gamma}} \cdot c_{e}^{\gamma}+e_{2, k}
\end{aligned}
$$

where $e_{1, k}$ and $e_{2, k}$ are the measurement errors with variance $S_{I}^{2}$ and $S_{G I R}^{2}$ for the insulin concentration and glucose infusion rate, respectively.

The two different types of insulin are injected as a subcutaneous bolus at $t=0$, i.e. $\int_{0^{-}}^{0^{+}} D d t=0.2 U / \mathrm{kgBW}$, while the other input variable in the model $R_{i n}$ is equal to $0.15 \mathrm{mU} / \mathrm{kg} \mathrm{BW} / \mathrm{min}$ throughout the experiment.

\section{Results and discussion}

The euglycaemic clamp data is modelled using the standard two-stage (STS) approach [3] where the individual parameters are assumed to be independent and identically distributed. A mixed-effect PK/PD modelling framework as implemented in NONMEM [1] is not considered since the theory for hierarchical PK/PD modelling using stochastic differential equations has not been developed. The following results are shown for a representative subject from the study along with the STS population parameters.

The PK/PD parameters in the effect-compartment model are estimated using only the observations at time instants where both insulin and GIR are observed. The parameters are first estimated for insulin B. Next, the estimated value of $E_{\text {max }}$ for insulin B is used as a fixed variable and the rest of the parameters are estimated for insulin A. This procedure is necessary for the estimation to converge for insulin A. Since the effect of the injected insulin does not come close to the maximum effect $E_{\text {max }}$ in this study, it is reasonable to assume that $E_{\text {max }}$ is the same for insulin A and $\mathrm{B}$ for the same subject.

The ML parameter estimates and their standard deviations for a representative subject from the study is shown in Table 1 for treatment with insulin A and B, respectively.

The limiting rate constant in the model is $k_{a}$ for insulin B since it is known that $k_{a}<k_{e}$ while the two rate constants are more equal for insulin A. When comparing the rate constants for absorption and elimination for the two types of insulin, $k_{a}$ seems to be the same while $k_{e}$ is about 5 times as large for insulin B than A. The change in the primary structure of insulin B compared to insulin A seems to alter the kinetics for elimination rather than that of absorption. This observation does not fit very well to the fact that it is $k_{a}$ which is attempted enlarged in insulin B to speed 
Table 1. PK/PD model parameter estimates for a representative subject from the study for treatment with insulin $\mathrm{A}$ and $\mathrm{B}$.

\begin{tabular}{|c|c|c|c|c|c|}
\hline \multirow[b]{2}{*}{ Parameter } & \multirow[b]{2}{*}{ Unit } & \multicolumn{2}{|c|}{ Insulin A } & \multicolumn{2}{|c|}{ Insulin B } \\
\hline & & $\hat{\theta}$ & SD & $\hat{\theta}$ & SD \\
\hline$q_{c, 0}$ & {$[\mathrm{nmol}]$} & $1.5886 E+01$ & $5.1881 E+00$ & $2.6384 E+00$ & $1.2955 E+00$ \\
\hline$c_{e, 0}$ & {$[\mathrm{nM}]$} & $7.3478 E-02$ & $2.3532 E-02$ & $1.0780 E-01$ & $3.1777 E-02$ \\
\hline$k_{a}$ & {$\left[\min ^{-1}\right]$} & $1.0776 E-02$ & $3.7624 E-03$ & $7.3497 E-03$ & $7.2944 E-04$ \\
\hline$k_{e}$ & {$\left[\min ^{-1}\right]$} & $7.8138 E-03$ & $2.5118 E-03$ & $3.9059 E-02$ & $6.6901 E-03$ \\
\hline$K_{e 0}$ & {$\left[\min ^{-1}\right]$} & $1.8323 E-02$ & $2.7653 E-03$ & $2.6053 E-02$ & $4.3714 E-03$ \\
\hline$V_{d}$ & {$[\mathrm{~L}]$} & $1.7744 E+02$ & $5.5831 E+01$ & $3.4359 E+01$ & $6.3678 E+00$ \\
\hline$\sigma_{s c}$ & {$[-]$} & $4.7650 E-11$ & $9.5767 E-06$ & $1.7460 E-07$ & $3.0731 E-02$ \\
\hline$\sigma_{c}$ & {$[-]$} & $1.5850 E-08$ & $2.4576 E-03$ & $7.2017 E-09$ & $1.4657 E-03$ \\
\hline$\sigma_{e}$ & {$[-]$} & $9.1515 E-10$ & $1.9153 E-04$ & $2.4896 E-12$ & $6.7938 E-07$ \\
\hline$E_{\max }$ & {$[\mathrm{mmol} / \mathrm{min}]$} & $9.2000 E+00$ & & $9.1570 E+00$ & $2.3370 E+00$ \\
\hline$E C_{50}$ & {$[\mathrm{nM}]$} & $3.0972 E-01$ & $1.6210 E-02$ & $2.6840 E-01$ & $7.7334 E-02$ \\
\hline$\gamma$ & {$[-]$} & $1.7554 E+00$ & $1.7843 E-01$ & $2.0325 E+00$ & $4.3342 E-01$ \\
\hline$S_{I}^{2}$ & {$[\mathrm{nmol} / \mathrm{L}]$} & $6.0129 E-04$ & $1.4430 E-04$ & $1.2529 E-03$ & $3.5428 E-04$ \\
\hline$S_{G I R}^{2}$ & {$[\mathrm{mmol} / \mathrm{min}]$} & $1.6574 E-03$ & $3.8409 E-04$ & $3.7529 E-03$ & $9.2274 E-04$ \\
\hline $\mathrm{t}_{\max }$ & {$[\mathrm{min}]$} & $1.0700 E+02$ & & $5.3000 E+01$ & \\
\hline $\mathrm{c}_{\max }$ & {$[\mathrm{pM}]$} & $3.2829 E+02$ & & $4.6584 E+01$ & \\
\hline $\mathrm{AUC}_{0}^{T}$ & [nM min] & $1.2070 E+02$ & & $1.2230 E+02$ & \\
\hline $\mathrm{TR}_{\max }$ & {$[\mathrm{min}]$} & $1.7100 E+02$ & & $1.0100 E+02$ & \\
\hline $\mathbf{R}_{\max }$ & {$[\mathrm{mmol} / \mathrm{min}]$} & $4.4300 E+00$ & & $6.3100 E+00$ & \\
\hline $\mathrm{GIR}_{0}^{T}$ & {$[\mathrm{~mol}]$} & $1.6600 E+00$ & & $1.8200 E+00$ & \\
\hline
\end{tabular}

up the absorption while no attempt is made to alter $k_{e}$. This phenomena is referred to as the 'flip-flop' effect. This effect is due to the fact that it is not always possible to separate $k_{a}$ and $k_{e}$ in the estimation. The 'flip-flop' effect can be circumvented by assuming that $k_{a}>k_{e}$, but since it is not possible to enter such assumptions in CTSM, the main difference between insulin A and B appears wrongly to be in the elimination kinetics. This is an example where the physiological knowledge, built into the grey-box modelling concept, is helpful.

The significantly different $V_{d}$ for the two types of insulin can partially be explained as the two types of insulin occupying a different volume in the body. The large and physiological unrealistic value of $V_{d}$ for insulin $\mathrm{A}$ is probably due to some of the insulin is bound in the tissue resulting in less insulin in the plasma. The volume is thereby overestimated since the model does not include a compartment for bound insulin or a bioavailability factor $F$ that compensates for non-available insulin.

The PD parameter for the maximal effect $E_{\text {max }}$ is the maximum glucose infusion rate (GIR). $E_{\max }$ is assumed to be the same for insulin A and B for the same subject since it is not possible to estimate $E_{\max }$ for insulin A. This is due to the 
fact that the data for insulin A only is distributed along the linear area of the sigmoidal curve in the range between $20 \%$ and $80 \%$ of $E_{\max }$. The estimated value of $E_{\max }=9.157 \mathrm{mmol} / \mathrm{min}$ for treatment with insulin B is therefore used as a fixed variable in the estimation for insulin A which is why no standard deviation is provided for that parameter. The estimated value of $E_{\max }$ is much higher than the value of the derived parameter $R_{\max }=6.3$ (maximum GIR) since the maximal effect is far from being reached.

The physical meaning of $E C_{50}$ is the insulin concentration producing $50 \%$ of the maximum effect. The parameter estimates of $E C_{50}$ for the two types of insulin are both around $0.3 \mathrm{nM}$ suggesting that the same insulin concentration of insulin $\mathrm{A}$ and $\mathrm{B}$ is needed to produce $50 \%$ of $E_{\text {max }}$.

The sigmoidicity parameter $\gamma$ is estimated close to 2 for both types of insulin. The theoretical meaning of the parameter $\gamma$ in the sigmoidal $E_{\max }$ model is that $\gamma$ insulin molecules and one receptor elicit the effect. The value of $\gamma \approx 2$ is normally also found in a static environment using in vitro cells exposed to insulin indicating that the simultaneous estimation of PK and PD parameters is reasonable.

The parameters describing the system noise $\sigma_{S C}, \sigma_{c}$, and $\sigma_{e}$ are all estimated to zero while the variance for the observation noise $S_{I}^{2}$ and $S_{G I R}^{2}$ are not, which indicates that the measured data does not deviate from the model except for the measurement error. The system noise parameters are the only parameters in the model which are not significantly different from zero on a $95 \%$ confidence level.

Persistently excited data is needed to be able to estimate all the parameters in a dynamical model. If the data are not excited enough, i.e. if the disturbance of the normal state of the system is not large enough in some sense to introduce (an) adequate dynamic state(s), it becomes difficult or impossible to estimate the parameters.

The time to maximum insulin concentration $t_{\max }$ for insulin $\mathrm{B}$ is almost half of that of insulin A while the maximum insulin concentration $c_{\max }$ is about $40 \%$ larger for insulin B than insulin A. The AUC is not very different for the two types of insulin and can therefore not be used to assess the difference in the treatment with insulin A and B. By comparing the three derived PK parameters it can be concluded that insulin B is a faster and shorter acting insulin compared to that of insulin A, while the amount of insulin absorbed throughout the study is almost the same for both types of insulin.

$T R_{\max }$ for both types of insulin occurs at a later time than $t_{\max }$ which is confirmed by the apparent delay between time to maximum insulin concentration and maximum response which results in a hysteresis loop. The glucose needed to maintain euglycemia throughout the study is not so different for the two types of insulin.

With the simultaneous estimation of PK and PD parameters, it is furthermore possible to access the correlation between the parameters shown in Table 1. The PK and PD parameters are not very correlated while the correlation between the PD parameters are quite high, especially between $E_{\max }$ and $E C_{50}$ where the correlation coefficient is estimated to 0.9853 .

The estimated insulin concentrations and GIR are shown in Fig. 4 along with a phase-plot of the observed GIR vs. the predicted effect compartment concentration. 


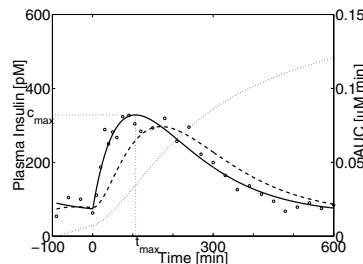

(a) PK A

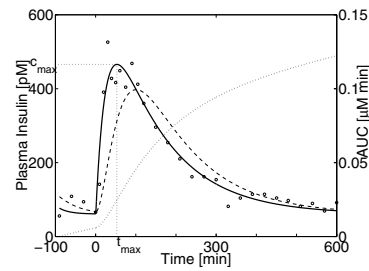

(d) PK B

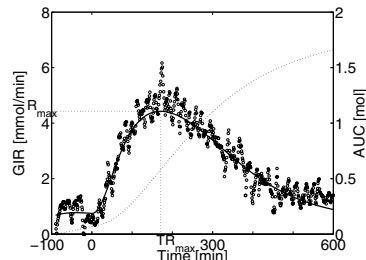

(b) GIR A

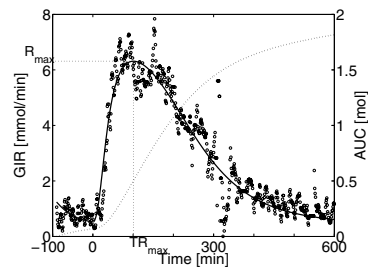

(e) GIR B

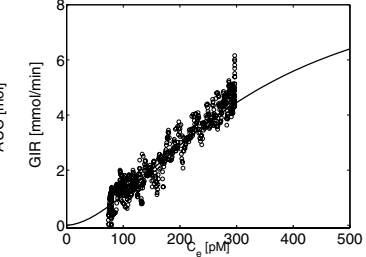

(c) Phase-plot A

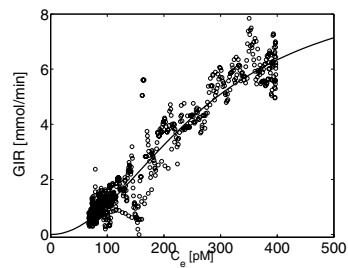

(f) Phase-plot B

Fig. 4. Plot of results for treatment with insulin A (Top) and insulin B (Bottom). (Left) Concentration-time profiles of measured (circle) and simulated (solid) plasma insulin concentration, effect compartment concentration (dashed), and AUC (dotted). (Middle) Measured (circle) and simulated (solid) GIR and AUC (dotted). (Right) Measured (circle) and simulated (solid) response.

The effect compartment concentration is slightly shifted towards the right compared with the plasma insulin concentration. The reason is that the insulin residing in the central compartment is not at steady-state resulting in the hysteresis loop shown in Fig. 2 while the effect compartment concentration is assumed to be at steady-state and thereby shifted to the right compared to the insulin in the central compartment.

The effect compartment concentration closes the hysteresis loop for both types of insulin. The relationship between the predicted effect compartment concentrations and GIR is very different because of the different estimated values of $E C_{50}$ and $\gamma$ which are influenced by the distribution time from the central to the effect compartment and the rate of insulin absorption.

The STS population parameters are calculated as the sample mean and standard deviation of the parameters of all twenty subjects in the study and shown in Table 2 for treatment with insulin A and B. Most of the STS population parameters are nonsignificant on a 5\% confidence level and the obvious next step in modelling the euglycaemic clamp would be to use a mixed-effects PK/PD modelling approach. Unfortunately, the theory for hierarchical grey-box PK/PD modelling has not yet been developed and it has therefore not been possible to explore this approach any further.

\section{Conclusion}

The proposed grey-box PK/PD modelling approach provides an effective method to estimate PK/PD parameters simultaneously, as well as the derived parameters 
Table 2. Standard two-stage population parameters for treatment with insulin A and B.

\begin{tabular}{llrlrrr}
\hline & & \multicolumn{2}{c}{ Insulin A } & & \multicolumn{2}{c}{ Insulin B } \\
\cline { 3 - 4 } \cline { 6 - 7 } \cline { 6 - 7 } Parameter Unit & \multicolumn{1}{c}{$\bar{\theta}$} & $\bar{s}$ & & $\bar{\theta}$ & $\bar{s}$ \\
\hline$q_{c, 0}$ & {$[\mathrm{nmol}]$} & 13.1847 & 8.0525 & & 4.3559 & 2.6807 \\
$c_{e, 0}$ & {$[\mathrm{nM}]$} & 0.0683 & 0.0432 & & 0.0558 & 0.0522 \\
$k_{a}$ & {$\left[\mathrm{~min}^{-1}\right]$} & 0.0090 & 0.0041 & & 0.0135 & 0.0061 \\
$k_{e}$ & {$\left[\mathrm{~min}^{-1}\right]$} & 0.0114 & 0.0074 & & 0.0248 & 0.0128 \\
$K_{e 0}$ & {$\left[\mathrm{~min}^{-1}\right]$} & 0.0303 & 0.0215 & & 0.0217 & 0.0086 \\
$V_{d}$ & {$[\mathrm{~L}]$} & 139.5820 & 53.9870 & & 66.3533 & 28.6696 \\
$\sigma_{s c}$ & {$[-]$} & 0.0000 & 0.0000 & & 0.0649 & 0.1685 \\
$\sigma_{c}$ & {$[-]$} & 0.0690 & 0.1538 & & 0.0728 & 0.1289 \\
$\sigma_{e}$ & {$[-]$} & 0.0006 & 0.0014 & & 0.0013 & 0.0037 \\
$E_{\text {max }}$ & {$[\mathrm{mmol} / \mathrm{min}]$} & 10.3 & 4.4 & & 10.3000 & 4.3630 \\
$E C_{50}$ & {$[\mathrm{nM}]$} & 0.4383 & 0.3181 & & 0.4267 & 0.3008 \\
$\gamma$ & {$[-]$} & 1.9790 & 0.7807 & & 1.7633 & 0.5974 \\
$S_{I}^{2}$ & {$[\mathrm{nmol} / \mathrm{L}]$} & 0.0008 & 0.0006 & & 0.0012 & 0.0005 \\
$S_{G I R}^{2}$ & {$[\mathrm{mmol} / \mathrm{min}]$} & 0.0028 & 0.0016 & & 0.0027 & 0.0016 \\
\hline
\end{tabular}

using all available information from the euglycaemic clamp study. It is furthermore possible to treat missing observations.

The effect-compartment model where the apparent delay between the plasma insulin concentration and the observed response is assumed to be distributional, is suitable for predicting the PD response with the Hill response equation as the effect model. The estimated PD parameters of the effect-compartment model are similar to those estimated from in vitro studies which is why the simultaneous estimation of PK and PD parameters is concluded to be successful.

The estimates of the diffusion term in the stochastic differential equations representing disturbances and unmodelled dynamics of the insulin/glucose system are insignificant from a statistical point of view in the three continuous time system equations. The proposed models therefore seem to capture the dynamics of the in vivo insulin/glucose system but it is difficult to make further conclusions since the experimental data from the considered clamp study is not persistently excited.

Acknowledgements. The authors wish to thank Novo Nordisk A/S for providing the data for the euglycaemic clamp study and Aage Vølund for helpful comments and suggestions with respect to PK/PD modelling of insulin.

\section{References}

1. Beal, S.L., Sheiner, L.B.: NONMEM User's Guides. NONMEM Project Group, University of California, San Francisco, 1994 
2. D'Argenio, D.Z., Park, K.: Uncertain pharmacokinetic/pharmacodynamic systems: design, estimation and control. Control Eng. Pract. 5 (12), 1707-1716 (1997)

3. Davidian, M., Giltinan, D.M.: Nonlinear Models for Repeated Measurement Data. Chapman \& Hall, London, 1995

4. Derendorf, H.: Pharmacodynamic Aspects of Systemic Drug Delivery. Drug Dev.Ind.Pharm. 20 (4), 485-502 (1994)

5. Kristensen, N.R., Melgaard, H., Madsen, H.: CTSM 2.0, User's Guide, 2001, http://www.imm.dtu.dk/CTSM.

6. Madsen, H., Holst, J.: Modelling Non-Linear and Non-Stationary Time Series. Informatics and Mathematical Modelling (IMM), DTU, 2000

7. Meibohm, B., Derendorf, H.: Basic Concepts of Pharmacokinetic/Pharmacodynamic (PK/PD) Modelling. Int. J. Clin. Pharm. Ther. 35 (10), 401-413 (1997)

8. Øksendal, B.: Stochastic Differential Equations - An Introduction with Applications. Springer-Verlag, Berlin, Germany, 5. edition, 1998

9. Pérez-Urizar, J., Granados-Soto, V., Flores-Murrieta, F.J., Castaneda-Hernández, G.: Review Article: Pharmacokinetic-Pharmacodynamic Modeling: Why? Arch. Med. Res. 31, 539-545 (2000)

10. Ramanathan, M.: An application of Ito's lemma in population pharmacokinetics and pharmacodynamics. Pharm. Res. 16 (4), 584-586 (1999)

11. Ramanathan, M.: Pharmacokinetic variability and therapeutic drug monitoring actions at steady state. Pharm. Res. 17 (5), 589-592 (2000)

12. Sheiner, L.B., Stanski, D.R., Vozeh, S., Miller, R.D., Ham, J.: Simultaneous Modelling of Pharmacokinetics and Pharmacodynamics: Application to d-tubocurarine. Clin. Pharmacol. Ther. 25, 358-371 (1979)

13. Tornøe, C.W.: Grey-box PK/PD Modelling of Insulin. Master's thesis. Informatics and Mathematical Modelling, DTU, 2002

14. Woodworth, J.R., Howey, D.C., Bowsher, R.R.: Establishment of Time-Action Profiles for Regular and NPH Insulin Using Pharmacodynamic Modeling. Diabetes Care 17 (1), 64-69 (1994)

15. Woodworth, J.R., Howey, D.C., Bowsher, R.R., Brunelle, R.L., Rowe, H., Compton, J., Cerimele, B.: Comparative Pharmacokinetics and Glucodynamics of Two Human Insulin Mixtures. Diabetes Care 17 (5), 366-371 (1994) 